\title{
Publisher's Note: Modular transformations through sequences of topological charge projections [Phys. Rev. B 94, 165108 (2016)]
}

Maissam Barkeshli and Michael Freedman

(Received 8 February 2017; published 17 February 2017)

DOI: 10.1103/PhysRevB.95.079904

This paper was published online on 5 October 2016 with an omission of an author affiliation. Michael Freedman's additional affiliation should read as "4 Department of Mathematics, University of California, Santa Barbara, California 93106, USA.” The additional author affiliation has been added as of 9 February 2017. The author affiliation is not present in the printed version of the journal. 